\title{
Versorgungsforschung - BRENDA und die Folgen
}

\author{
A. Wöckel, R. Kreienberg (Leitlinienkoordinator der S3-Leitlinie „Diagnostik, Therapie und Nachsorge des Mammakarzinoms“ \\ und 2. Vizepräsident der Deutschen Gesellschaft für Gynäkologie und Geburtshilfe)
}

\section{Leitlinien als Grundlage \\ einer Versorgungsoptimierung}

Trotz kontinuierlicher therapeutischer Verbesserungen stellt das Mammakarzinom ein gesundheitspolitisch und -ökonomisch relevantes Versorgungsproblem dar. Um eine evidenzbasierte Versorgung zu ermöglichen, wurde bereits 2004 die erste nationale „S3-Leitlinie zur Diagnostik, Therapie und Nachsorge des Mammakarzinoms“ unter der Federführung der Deutschen Krebsgesellschaft (DKG) entwickelt. Nach den Kriterien der evidenzbasierten Medizin (EbM) erfolgte eine systematische Recherche und im Anschluss eine Selektion der klinisch und methodisch relevanten Studien, bevor aus den extrahierten Ergebnissen nach Konsensusprozessen entsprechende Empfehlungen und Statements formuliert werden. Damit beruht die erste S3-Leitlinie zur Behandlung des Mammakarzinoms und deren erste Aktualisierung von 2008 auf der derzeit best verfügbaren Evidenz und den zugrunde liegenden methodisch hochwertigen Studien. Im Umkehrschluss ist jedoch unklar, ob durch die Anwendung der Leitlinienempfehlungen (leitlinienkonformes Verhalten) bestimmte Outcomes des definierten Versorgungsproblemes, wie z.B. rezidivfreies Überleben oder auch Gesamtüberleben, überhaupt verbessert werden können, da Studien zu dieser Frage in der Versorgungsforschung bisher kaum durchgeführt wurden.

\section{Das BRENDA-Projekt als Meilenstein der onkologischen Versorgungsforschung $\nabla$}

In einer Kohortenstudie wurden Patientinnen mit primärem Mammakarzinom im Rahmen des BRENDA-Projektes („Breast Cancer Care Under Evidence-based Guidelines“) analysiert, die an der Universitätsfrauenklinik Ulm und 16 benachbarten Netzwerkkliniken therapiert wurden. Projektpartner für die umfangreichen statistischen Analysen sind die Arbeitsgruppen von Prof. M.Wischnewsky, Universität Bremen und Frau Prof. M. Blettner, Universität Mainz. Ziele der Studie waren die deskriptive Beschreibung der Leitlinienkonformität bei der Therapie des Mammakarzinoms im zeitlichen Verlauf sowie die Darstellung patientenrelevanter Outcomes (Gesamtüberleben (OAS) und Rezidivfreies Überleben (RFS)) beim Einhalten von Leitlinienempfehlungen. Leitlinienkonformität wurde nach dieser systematischen Analyse der individuellen Krankengeschichte nach den Leitlinienempfehlungen und Statements der S3-Leitlinie (Version 2004) definiert. In dem Modell war Leitlinienkonformität dann gegeben, wenn in der Bewertung in keinem Fall ein Abweichen von Empfehlungen vorlag. In diesem vom BMBF geförderten und laufenden Förderprojekt BRENDA I konnte gezeigt werden, dass Leitlinienkonformität in der adjuvanten Situation sowohl im unizentrischen Ansatz (Universitätsfrauenklinik Ulm 1992-2005) als auch im multizentrischen Vergleich (16 angrenzende Netzwerkkliniken bzw Brustzentren 2003-2005) zu signifikant besseren Rezidivfreien- und Gesamtüberlebensraten führte.
Dies gilt in den bisherigen Auswertungen für alle Altersgruppen, für alle Tumorstadien und für alle Risikokonstellationen. Bisher konnten die Datensätze von über 10000 Patientinnen mit primärem Mammakarzinom ausgewertet werden: Ein wichtiges Ergebnis des BRENDA-I-Projektes ist, dass sich die Leitlinienverletzungen bezüglich Primäroperation, Axilladissektion, der Strahlentherapie sowie des Einsatzes einer Chemo- und / oder Hormontherapie hinsichtlich der Reduktion des krankheitsfreien Überlebens und des Gesamtüberlebens unterschiedlich auswirken, insgesamt sich jedoch bei Mehrfachverletzungen zu einer erheblichen Ergebnisreduktion addieren. Die Analyse zeigte, dass die Behandlungsgruppe „Nicht Leitlinienkonforme Therapie“ primär eine hochsignifikant schlechtere Prognose (Nottingham Prognose Index NPI =4,56) aufweist, als die Behandlungsgruppe „Leitlinienkonforme Therapie“ (NPI=4,07), d. h. Patientinnen mit höherem Risiko wurden zu einem höheren Anteil nicht-leitlinienkonform behandelt als Patientinnen mit niedrigerem Risiko.

\section{Welche Patientinnen werden nicht leitlinienkonform therapiert? \\ $\nabla$}

Von besonderer Bedeutung ist die Tatsache, dass die maximale erreichbare Leitlinienkonformität seit 2001 bei ca. 60 bis $70 \%$ konstant geblieben ist und sich auch bis heute nur marginal geändert hat. Dies legt die Vermutung nah, dass nicht die Leitlinien-Implementierung, sondern Barrierefaktoren von Seiten der Ärzte und/oder der Patientinnen für die mangelnde Leitlinienkonformität verantwortlich sind. Eine Analyse dieser Barrierefaktoren, die für die Frage von entscheidender Bedeutung ist, ob die Leitlinienverletzung im Umfang von 40\% hingenommen werden muss, oder ob durch Interventionen auf Arztund Patientenseite diese zu überwinden sind, werden in einem Folgeprojekt BRENDA II prospektiv in einem Multizenter-Ansatz untersucht, um die die Barrierefaktoren zu charakterisieren, die zur Modifikation der therapeutischen Schritte (Leitlinienverletzung) geführt haben.

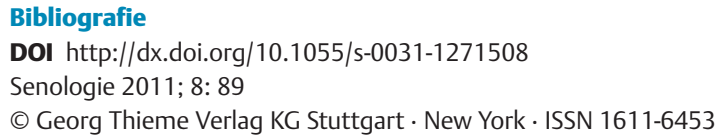

\section{Korrespondenzadressen}

PD Dr. med. Achim Wöckel

Universitätsfrauenklinik Ulm Prittwitzstr. 43

89075 Ulm

achim.woeckel@uniklinik-ulm.de

\section{Prof. Dr. med. Rolf Kreienberg}

Ärztlicher Direktor der Universitätsfrauenklinik Ulm

Prittwitzstr. 43

89075 Ulm

rolf.kreienberg@uniklinik-ulm.de 